\title{
Conducta anestésica en el embolismo aéreo, durante la cirugía de fosa posterior con posición sedente, en el paciente pediátrico
}

Pascual PM, Ferreras R, Romero M, Jiménez L, Rubio P.

Hospital 12 de Octubre, Madrid.

\section{Resumen}

El embolismo aéreo constituye una situación de emergencia en neurocirugía pediátrica. Presenta una incidencia entre el 9,8 y $0,42 \%$. Uno de los factores de riesgo más relevantes para su aparición es la posición quirúrgica sedente, necesaria en muchas ocasiones para poder realizar procedimientos quirúrgicos sobre fosa craneal posterior.

\section{Introducción}

El embolismo aéreo constituye una situación de emergencia en neurocirugía pediátrica. Presenta una incidencia entre el 9,8 y $0,42 \%(1,2)$. Uno de los factores de riesgo más relevantes para su aparición es la posición quirúrgica sedente, necesaria en muchas ocasiones para poder realizar procedimientos quirúrgicos sobre fosa craneal posterior (3).

\section{Descripción}

Se trata de un paciente de 11 años y 50 $\mathrm{kg}, \quad \sin$ antecedentes destacables ni alergias, que acude al hospital con clínica de emesis de repetición y de focalidad neurológica en forma de marcha en tándem (Romberg negativo) y disartria. Se realiza una resonancia magnética objetivándose una lesión ocupante de espacio en vermis cerebeloso de característica quísticonodular, con predominio quístico, junto con hidrocefalia supratentorial. Con todo ello se decide realizar exéresis tumoral de forma programada.

Previo a la cirugía, se solicitó el preoperatorio con una analítica (hemograma, coagulación y bioquímica básica), pruebas cruzadas, $y$ un ecocardiograma con test de burbujas. El objetivo de dicho ecocardiograma es descartar la presencia de defectos septales que puedan suponer la contraindicación de la posición en sedestación (4) debido al incremento del riesgo asociado a embolismo aéreo. Todas las pruebas resultaron dentro de la normalidad, siendo el paciente apto para la intervención en la posición convenida.

El paciente acudió a quirófano con Glasgow 15 estable hemodinámica y respiratoriamente. Antes de la inducción, se procedió a la monitorización con NIRS, BIS, pulsioximetría periférica, electro cardiograma (ECG) y tensión arterial no invasiva. A continuación, se realizó preoxigenación con mascarilla facial con $\mathrm{FiO}_{2}$ 1, e Inducción intravenosa con (fentanilo $3 \mathrm{mcg} / \mathrm{kg}$, propofol $3 \mathrm{mg} / \mathrm{kg}$ y 
rocuronio $0.6 \mathrm{mg} / \mathrm{kg} \mathrm{mg).} \mathrm{Se} \mathrm{procedió} \mathrm{a}$ intubación orotraqueal con tubo flexometálico número 6.5 bajo laringoscopia directa, sin que se produjese ninguna incidencia. Se conectó a ventilación mecánica controlada por presión con una fracción inspiratoria de oxígeno $\left(\mathrm{FiO}_{2}\right)$ de 0.35 , presión positiva al final de la espiración (PEEP) de $5 \mathrm{~cm}$ de $\mathrm{H}_{2} \mathrm{O}$, presión inspiratoria de $13 \mathrm{~cm}$ de $\mathrm{H}_{2} \mathrm{O}$ y frecuencia respiratoria de 15 respiraciones por minuto.

Posteriormente, se canalizó la vena yugular interna derecha ecoguiada (7 fr), la arteria radial izquierda (22 fr), se colocó una sonda nasogástrica del $\mathrm{n}^{\mathrm{o}} 12$, y una sonda vesical. El mantenimiento anestésico se realizó con perfusión de propofol y remifentanilo y bolos de fentanilo. Durante la cirugía también fueron monitorizados potenciales sensitivos y motores por neurofisiólogo.

La profilaxis antibiótica se llevó a cabo con cefazolina $25 \mathrm{mg} / \mathrm{kg}$ previa a la incisión quirúrgica, aplicando una dosis de refuerzo a las 4 horas de la intervención. Antes del cambio de posición supina a sedestación, se realizó un vendaje compresivo de miembros inferiores (5) y se protegieron debidamente las zonas proclives; mientras se administraba un bolo con solución balanceada isotónica a 15 $\mathrm{ml} / \mathrm{kg}$. A pesar de ello, tras colocar al paciente en posición sedente o Fowler, se produjo un descenso de un $30 \%$ en la presión arterial media por lo que se continuó con fluidoterapia intensiva ente $7-6 \mathrm{ml} / \mathrm{h}$ durante las siguientes dos horas.

En el transcurso del intraoperatorio, se produjeron dos embolismos aéreos, ambos con PVC en torno a $7 \mathrm{~cm}$ de $\mathrm{H}_{2} \mathrm{O}$. El primero ocurrió a las dos horas de la incisión quirúrgica, comenzando con un descenso brusco del $\mathrm{EtCO}_{2}$ de 33 a $27 \mathrm{mmHg}$. Al cabo de un minuto aproximadamente del anterior descenso, comenzó una desaturación de $99 \%$ a $74 \%$ con una taquicardia sinusal a 125 lpm. Minuto y medio después, se objetivó una caída del NIRS de 80 a 63 (Figura 3). Por último, se produjo un descenso de presión arterial de 85/40 hasta $54 / 35 \mathrm{mmHg}$ parejo a una pérdida de potenciales motores y sensitivos, con una diferencia de aproximadamente 2 minutos y medio desde la caída inicial del etCO $\mathrm{C}_{2}$.

Tras el inicio de la desaturación, se procedió inmediatamente a la aspiración de burbujas a través de la luz distal de la vía venosa central (Figura 1). Al mismo tiempo, se informó a los neurocirujanos del episodio, por lo que estos comenzaron a irrigar el campo quirúrgico con suero para, posteriormente, taparlo con compresas húmedas. Por parte del equipo de anestesia se aumentó el $\mathrm{FiO}_{2}$ a 1 y se administró un bolo de coloide de 5 $\mathrm{ml} / \mathrm{kg}$. En menos de 5 minutos se recuperaron los parámetros hemodinámicos, respiratorios $\mathrm{y}$ potenciales sensitivo-motores previos. La gasometría de control posterior al episodio mostró una hemoglobina de $12.5 \mathrm{~g} / \mathrm{d}$ l, y un láctico de 1.6 (previo de 0.9 ), quedando el resto de parámetros dentro de la normalidad.

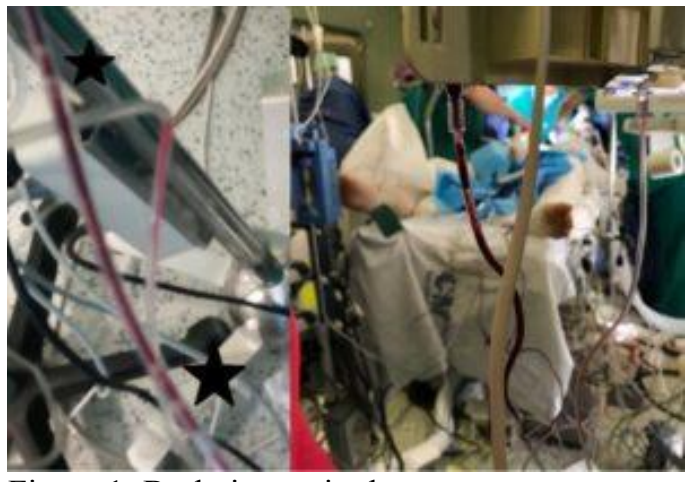

Figura 1: Burbujas aspiradas

El segundo episodio ocurrió aproximadamente con dos horas $y$ media de diferencia respecto al anterior. 
La resección tumoral había concluido y los neurocirujanos se encontraban revisando el lecho quirúrgico. Los cambios en la monitorización siguieron la misma cronología que en el episodio anterior con una caída de $\mathrm{EtCO}_{2}$ de 35 a $17 \mathrm{mmHg}$, desaturación de $99 \%$ hasta $84 \%$, taquicardia sinusal a $125 \mathrm{lpm}$, caída de NIRS (Figura 3), e hipotensión (de $80 / 50 \mathrm{mmHg}$ a $48 / 30 \mathrm{mmHg}$ ) con pérdida de los potenciales motores $\mathrm{y}$ sensitivos. Tras aproximadamente un minuto y medio de la hipotensión, aparecen cambios electrocardiográficos consistentes en un descenso del intervalo ST, seguido de un ascenso (Figura 2), solamente objetivado en las derivaciones II y III, por motivos de la urgencia del evento. En ese momento se decidió el cambio de posicionamiento de sedestación a posición en decúbito supino al paciente. Asimismo, se observaron pupilas isocóricas durante este episodio.

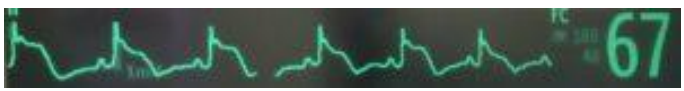

Figura 2: Ascenso ST observado en monitor (derivación II)

Las medidas tomadas en este segundo episodio fueron: irrigación de campo con suero, aumento de $\mathrm{FiO}_{2}$ a 1, bolo de coloide de $5 \mathrm{ml} / \mathrm{kg}$, fenilefrina (dos bolos, uno de $20 \mathrm{mcg}$ y otro de $50 \mathrm{mcg}$ ), aspiración de grandes cantidades de burbujas $\mathrm{y}$, finalmente, cambio de posicionamiento de la paciente a posición supina. Tras el cambio de posición se logró estabilizar hemodinámicamente al paciente, revirtiendo los cambios respiratorios y electrocardiográficos. Sin embargo, no se recuperaron totalmente los potenciales motores derechos. Una vez confirmada la estabilidad hemodinámica, se colocó en decúbito prono para el cierre de la incisión quirúrgica. No hubo alteraciones significativas en la gasometría realizada posteriormente, salvo el láctico, de 1.9 $\mathrm{mmol} / \mathrm{L}$.

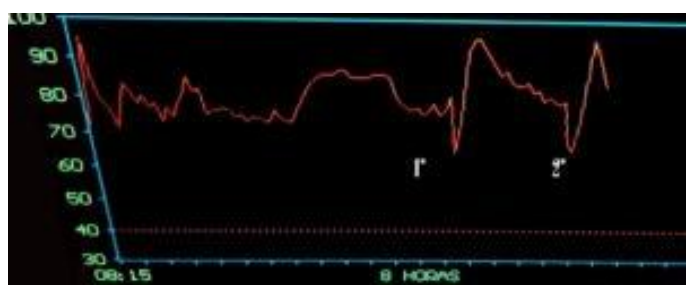

Figura 3: Imagen NIRS: $1 *$ y $2 *$, descenso en los valores del NIRS en cada episodio de embolismo aéreo.

Fuera de estos dos episodios, el paciente mantuvo tensión arterial media entre $56-60 \mathrm{mmHg}$, NIRS estable en torno a 80-85, y $\mathrm{SpO}_{2}$ de $99 \%$.

No precisó hemoderivados en el intraoperatorio.

Al finalizar el procedimiento, se trasladó al paciente a la UCI de anestesia pediátrica en ventilación mecánica $\left(\mathrm{FiO}_{2}: 0.35 \%\right.$, PEEP 5, Pins 13, FR 15), sedada con Remifentanilo $(0.05 \mathrm{mcg} / \mathrm{kg} / \mathrm{min}) \quad$ y $\quad$ Propofol $(4 \mathrm{mg} / \mathrm{kg} / \mathrm{h})$.

Tras su ingreso en el postoperatorio inmediato, se objetivaron dos episodios de bradicardia hasta $35 \mathrm{lpm}$, sin repercusión hemodinámica. Se realizó un electrocardiograma en el que se encontró bradicardia sinusal a $451 \mathrm{pm}$, eje QRs normal. PR constante, no bloqueo. QTc prolongado. Repolarización anormal con aplanamiento generalizado de ondas $\mathrm{T}$. Además, se solicitó ecocardiograma en el que se objetivó el ventrículo derecho con disfunción sistólica en global leve moderada (TAPSE $15 \mathrm{~mm}$ ).

La analítica de control postoperatorio mostró: CK 229 U/l, Troponina T hs $118.0 \mathrm{ng} / \mathrm{l}$, hemoglobina $12,3 \mathrm{~g} / \mathrm{dl}$ como datos destacables. 
Figura 4: Placa de tórax donde se observa VVC en VCS, a pesar de lo cual se consiguió aspirar las burbujas.

Durante su estancia en la UCI de anestesia pediátrica presentó una evolución favorable.

Tras comprobar la estabilidad hemodinámica y con PAFi $>300$, se retiró la sedación, y se llevó a cabo el destete de la ventilación mecánica, manteniéndose eupneica y con buenas saturaciones tras la extubación. Desde el punto de vista hemodinámico, tenía tendencia a la bradicardia durante las primeras $24 \mathrm{~h}$ postoperatorias sin repercusión clínica ni hemodinámica.

Tras la extubación, la exploración neurológica inicial mostró bradipsiquia, disminución de tono en miembro inferior izquierdo, ausencia de tono en miembro superior izquierdo con movilización espontánea de miembros derechos. Reflejos conservados.

Se realizó un TAC craneal donde se observaron campos postquirúrgicos en fosa posterior con escasos restos hemáticos, pero con abundante neumoencéfalo de predominio bifrontal.

Progresivamente, durante las primeras 24 horas, recuperó movilidad de las extremidades izquierdas. En el ecocardiograma de seguimiento a las $24 \mathrm{~h}$ se comprobó la recuperación completa de la función sistólica del ventrículo derecho. Se realizó una seriación de las enzimas de daño miocárdico con valores pico de $\mathrm{Ts}$ $118 \mathrm{mg} / \mathrm{L}$ y descenso posterior hasta normalización.

En el ECG a su salida de REA presentaba: Repolarización anormal con aplanamiento generalizado, $\mathrm{T}$ negativas en precordiales $y$ algunas de las derivaciones frontales, aunque sin alteraciones del ST, ni ondas Q patológicas.

Fue trasladada a planta de hospitalización a las 72 horas del ingreso.

\section{Discusión}

La posición sedente durante una cirugía de fosa posterior es utilizada para un mejor abordaje quirúrgico así como para disminuir el sangrado a lo largo de la intervención. En pacientes sometidos a anestesia general, favorece la ventilación con respecto a la posición supina, produciéndose menos atelectasias y efecto shunt (3). Sin embargo, tiene importantes efectos hemodinámicos como el aumento de la frecuencia cardíaca y de las resistencias vasculares, y el descenso de presión arterial, el retorno venoso y el gasto cardíaco. Entre los riesgos conocidos está la posibilidad de sufrir un embolismo aéreo, debido a la exposición de los sinusoides venosos del hueso por encima del nivel del corazón. Cuando la diferencia de presiones entre la aurícula y los senos venosos supera los $5 \mathrm{~cm}$ de agua (6), el aire puede pasar a la circulación pulmonar $\mathrm{y}$ generar un fracaso del ventrículo derecho. La mayor parte de los embolismos aéreos son asintomáticos; sin embargo, un volumen de aire entre $5-6 \mathrm{ml} / \mathrm{kg}$ es letal (7). 


\section{Diagnóstico}

El diagnóstico habitual se basa en descenso de etCO $\mathrm{O}_{2} \mathrm{e}$ hipotensión no explicable por otras causas. La ecografía transesofágica es la prueba más sensible; sin embargo, si no hay descenso del etCO $\mathrm{C}_{2}$, la probabilidad de que el embolismo aéreo cause alteraciones hemodinámicas es muy baja (8). La cronología expuesta (Figura 5) demuestra que antes de que se produzca hipotensión hay pruebas no invasivas cuya alteración es más precoz. El NIRS tiene la ventaja de ser una monitorización no invasiva, fácil de aplicar y representada por una tendencia numérica, por lo que las alteraciones son fáciles de evidenciar.

\section{Prevención y tratamiento}

La prevención se lleva a cabo con la optimización de la PVC; sin embargo, no elimina el riesgo de embolismo aéreo.

Informar al cirujano del evento es fundamental debido a que la irrigación con suero puede ser una de las medidas más efectivas junto con el mantenimiento hemodinámico (hidratación y, si es necesario, el uso de drogas vasoactivas).

Aumentar fracción inspiratoria de oxígeno mejora el intercambio gaseoso y favorece la eliminación de nitrógeno.

Cambiar la posición de la cabeza, si es posible, y aspirar las burbujas son medidas probadas.

El uso de presión positiva al final de la espiración es controvertido (9). En cuanto al cambio de posicionamiento, la cabeza del paciente se debe reposicionar a nivel del corazón y, si es posible, usar el decúbito lateral izquierdo para facilitar el paso de las burbujas a la aurícula derecha. En el segundo evento, esa decisión fue marcada por el empeoramiento clínico progresivo a pesar de las medidas tomadas.

Debido a que se trata de un empeoramiento rápido, cuya reversión también puede tener lugar en minutos, es difícil saber el momento para el cambio de la posición. Además, el momento de desarrollo en que se encuentre la cirugía puede suponer un obstáculo más para el reposicionamiento a supino.

La isquemia miocárdica secundaria a embolismo aéreo es una situación grave que requiere de una valoración de su repercusión mediante un ecocardiograma, donde se valorará el estado de la contractilidad; un electrocardiograma y un seguimiento de enzimas de daño miocárdico.

Las alteraciones neurológicas deben descartarse lo antes posible realizando una ventana de sedación o extubación, si la situación lo permite, y realizándose pruebas de imagen si la evolución no es favorable.

El neumoencéfalo postoperatorio puede llegar a tener una incidencia del 100\% (3) en los pacientes que han sido intervenidos en esta posición, debido a la presión negativa del LCR y al paso de aire durante el cierre de la duramadre.

\section{Conclusiones}

La hidratación previa y optimización de los valores de PVC no elimina el riesgo de aparición de embolismo aéreo, por lo cual el diagnóstico precoz es fundamental. El etCO ${ }_{2}$ es una prueba muy sensible y fácil de interpretar. El NIRS no es invasivo y nos da información sobre el estado hemodinámico antes que el descenso de la presión arterial. Es fundamental la comunicación con los padres explicando lo sucedido de manera clara $\mathrm{y}$ 
entendible además de advertir de las posibles secuelas.

\section{Bibliografía}

1. Aleksic V, Radulovic D, Milakovic B, Nagulic M, Vucovic D, Antunovic V, et al. A retrospective analysis of anesthesiologic complications in pediatric neurosurgery. Paediatr Anaesth. 2009 Sep;19(9):879-86. (PubMed) (Abstract)

2. Harrison EA, Mackersie A, McEwan A, Facer E. The sitting position for neurosurgery in children: a review of 16 years' experience. $\mathrm{Br} \mathrm{J}$ Anaesth. 2002 Jan;88(1):12-7. (PubMed)

3. Palmon SC, Moore LE, Lundberg J, Toung T. Venous air embolism: a review. J Clin Anesth. 1997 May;9(3):251-7. (Pubmed)

4. Standefer M, Bay JW, Trusso R. The sitting position in neurosurgery: a retrospective analysis of 488 cases. Neurosurgery. 1984 Jun;14(6):649-58. (PubMed)

5. Rozet I, Vavilala MS. Risks and Benefits of Patient Positioning During Neurosurgical Care. Vol. 25, Anesthesiology clinics. 2007. p. 631-X. (HTML) (PDF)

6. Shaikh N, Ummunisa F. Acute management of vascular air embolism. J Emerg Trauma Shock. 2009 Sep;2(3):180-5. (PubMed) (HTML)
7. Toung TJ, Rossberg MI, Hutchins GM. Volume of air in a lethal venous air embolism. Anesthesiology. 2001 Feb;94(2):360-1. (PubMed) (HTML)

8. Pandia MP, Bithal PK, Dash HH, Chaturvedi A. Comparative incidence of cardiovascular changes during venous air embolism as detected by transesophageal echocardiography alone or in combination with end tidal carbon dioxide tension monitoring. J Clin Neurosci Off J Neurosurg Soc Australas. 2011 Sep;18(9):12069. (PubMed) (HTML)

9. Giebler R, Kollenberg B, Pohlen G, Peters J. Effect of positive endexpiratory pressure on the incidence of venous air embolism and on the cardiovascular response to the sitting position during neurosurgery. $\mathrm{Br} \mathrm{J}$ Anaesth. 1998 Jan;80(1):30-5. (PubMed) ( $\underline{\text { PDF) }}$

Correspondencia al autor

Patricia María Pascual Cambero patrypc_3@hotmail.com FEA Adjunta de Anestesiología y Reanimación pediátrica.

Hospital 12 de Octubre, Madrid

Aceptado para blog en mayo de 2018. 